\title{
Estimation of the heat of absorption of CO2 by IR imaging of the surface of solid absorbents.
}

by F.Benevides* ${ }^{\star}$ Christophe Pradère ${ }^{\star}$, Jean-Christophe Batsale*, Gwenaelle Le Bourdon ${ }^{\star *}$, Joelle Mascetti ${ }^{* *}$, Laurent Servant $^{\star *}$, Julien Jolly ${ }^{\star \star *}$, Bertrand Pavageau ${ }^{\star \star \star}$

* Univ. Bordeaux, I2M, UMR 5295, F-33400 Talence, France, c.pradere@i2m.u-bordeaux1.fr

** Univ. Bordeaux, ISM, UMR 5255, F-33400 Talence, France, l.servant@ism.u-bordeaux1.fr

*** Univ. Bordeaux, LOF, UMR 5258,F-33400 Talence, France, Julien.JOLLY@solvay.com

\section{Abstract (Arial, 9pt, bold)}

The mechanisms of $\mathrm{CO}_{2}$ capture by solid adsorbents has been investigated. The understanding of physicochemical mechanisms controlling $\mathrm{CO}_{2}$-adsorbent interactions is primordial for the choice of materials and for the design of industrial capture processes. A key parameter used in these investigations is the heat released during the adsorption of the gas on the adsorbent. Thanks to infrared thermography and an analytical balance, we obtained the estimation of the $\mathrm{CO}_{2}$ adsorption heats on several types of porous materials. This work describes the device and the IR image processing employed to the estimation of the heats of adsorption.

\section{Introduction}

Extensive efforts have been devoted to reduce $\mathrm{CO}_{2}$ emissions to fight against the related increase in global average temperatures since the mid-20th century [1]. One of the most investigated solutions is the development of $\mathrm{CO}_{2}$ capture technologies [2]. The process of absorption of gaz charged with $\mathrm{CO}_{2}$ through a porous solid absorbent is one of them.

The adsorption of a gas on an adsorbent is exothermic. The study of the transient energy released during $\mathrm{CO}_{2}-$ adsorbent interaction, leads to a better understanding of these physicochemical mechanisms. The estimation of the transient adsorption heat is thus crucial. A high adsorption heat leads to a high $\mathrm{CO}_{2}$-adsorbent interaction. This means that the material has a good affinity with the $\mathrm{CO}_{2}$ molecules, allowing favorable $\mathrm{CO}_{2}$ capture. However, energetic interactions may impede the material regeneration (desorption of $\mathrm{CO}_{2}$ from adsorbent). Therefore, the estimation of adsorption heat is a key parameter for the selection of materials. This work presents a high-throughput device based on infrared thermography coupled to a precision balance to in-situ estimations of adsorption heats via a thin-layer approach. A simplified heat transfer model and the general approach employed for the estimation of transient adsorption heats, using an inverse technique, are then presented.

\section{Experimental device}

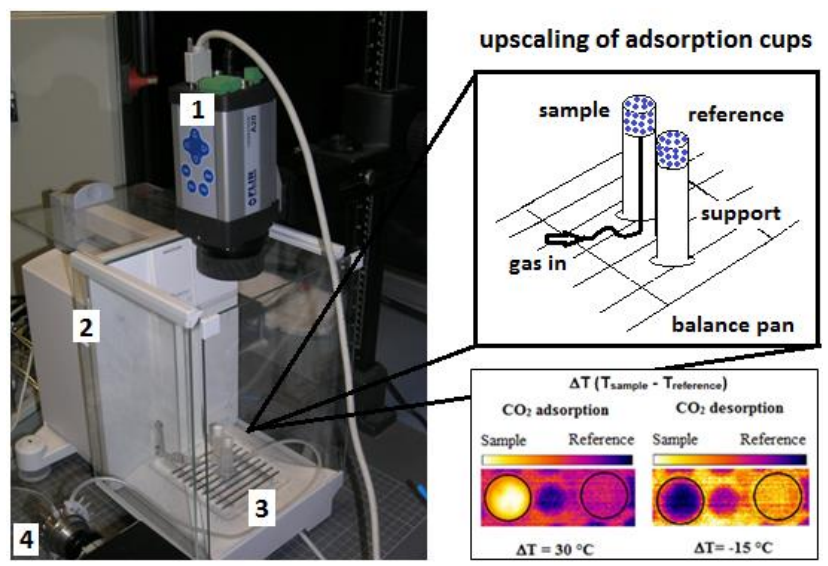

Figure 1. Picture of the high-throughput experimental device, scheme of thin-layer configuration and infrared images of adsorption/desorption process. 
One infrared camera FLIR System A20M (Figure 1. number 1) was coupled with an analytical balance METTLER TOLEDO XS204 DeltaRange (Figure 1: number 2) for simultaneous recording of the average temperature and mass variation of a thin-layer of adsorbent during $\mathrm{CO}_{2}$ adsorption and desorption at room temperature. The sample mass variation is associated with adsorbed-phase evolution during $\mathrm{CO}_{2}$ adsorption/desorption. The IR camera allows the detection of thermal radiation (from 7.5 to $13 \mu \mathrm{m}$ ) coming from the adsorbent surface, and the temperature values can be directly measured through calibration parameters. A differential temperature measurement is recorded (sampling rate $=$ $11 \mathrm{~Hz}$ ) by using the same material as a reference to take into account the material emissivity and room temperature variation during the experiment. The precision balance measures the sample mass, and thanks to home-made Labview interface, the sample mass evolution is recorded (sampling rate $=1 \mathrm{~Hz}$ ). Two filtering cartridges (made of insulating material) filled with a thin-layer of adsorbent (from 1 to $3 \mathrm{~mm}$ ) are placed and fixed on the balance scale pan (Figure 1: number 3 ) and one of them is connected with a gas injection system (Figure 1: number 4). The injection system (multiposition valve, mass flow meters and 3-way valves) allows the switch on either $\mathrm{CO}_{2}$ gas or $\mathrm{N}_{2}$ gas (inert gas used to study $\mathrm{CO}_{2}$ desorption) with a controlled injection time (home-made Labview interface). The 3-way valves are used to prevent excessive pressure between gas switching, thus the flow rate can be controlled without variation during adsorption/desorption cycles. This device allows thus fast, non-contact and in-situ measurements as a function of a specific experimental protocol. The use of an infrared camera allows to estimate simultaneously several samples and to analyse the uniformity of the adsorption process.

\section{Experimental results}

From the previous device, the figure 2 shows an example of simultaneous recording of temperature and mass variation of a thin-layer of adsorbent during $\mathrm{CO}_{2}$ adsorption and desorption cycles at room temperature $\left(600 \mathrm{~s}\right.$ of $\mathrm{CO}_{2}+$ $600 \mathrm{~s}$ of $\mathrm{N}_{2}$, flow rate $=40 \mathrm{ml} \cdot \mathrm{min}^{-1}$, thin-layer $=3 \mathrm{~mm}$ ). We notice that the flow of $\mathrm{CO}_{2}$ gas through thin-layer have led to fast elevation of temperature followed by mass uptake. On the contrary, the flow of $\mathrm{N}_{2}$ gas through thin-layer have led to fast decrease of temperature flowed by loss of mass. These results characterize the adsorption and desorption of $\mathrm{CO}_{2}$ molecules on/from adsorbent surface respectively.

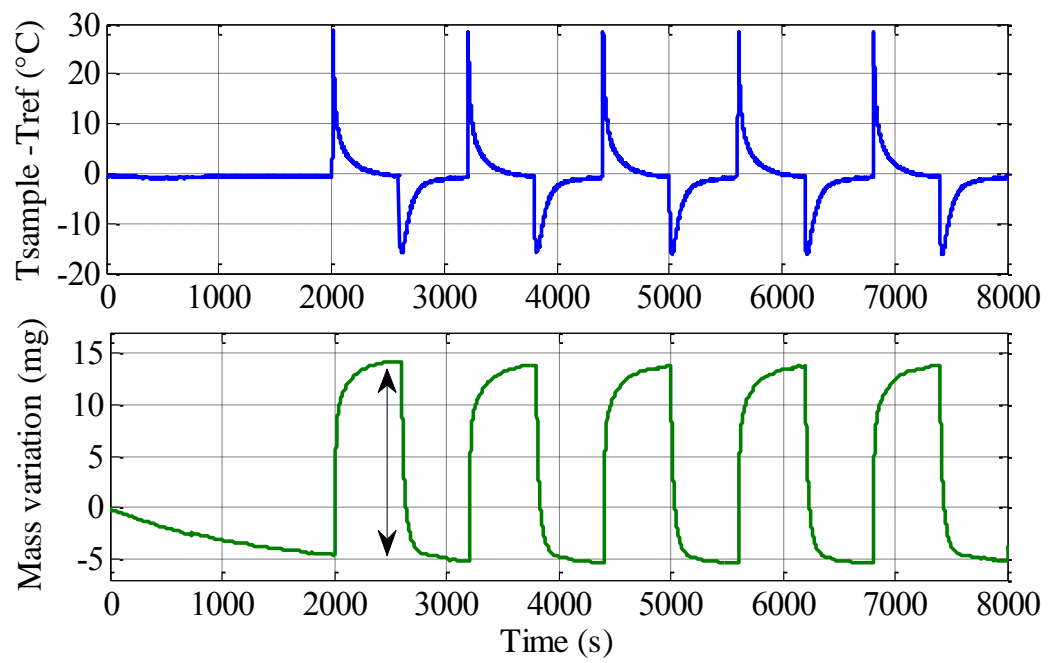

Figure 2. Evolution of mean surface temperature and sample mass during $\mathrm{CO}_{2} / \mathrm{N}_{2}$ injection cycles through a thin-layer of an activated carbon at room temperature.

The main originality of this study compared to a previous preliminary study (see [3]) is that the experimental results will be processed by considering simultaneously the transient temperature signal and the mass variation of the sample. The transient energy balance will be considered with the lumped temperature of the sample and a simultaneous estimation of the heat losses.

\section{REFERENCES}

[1] Plasseraud L. Carbon Dioxide as Chemical Feedstock. Edited by Michele Aresta. ChemSusChem. 2010;3:6312.

[2] Figueroa JD, Fout T, Plasynski S, Mcllvried H, Srivastava RD. Advances in CO2 capture technology-The U.S. Department of Energy's Carbon Sequestration Program. International Journal of Greenhouse Gas Control. 2008;2:9-20.

[3] Jolly J, Pavageau B, Tatibouët J-M. Time-resolved IR thermographic detection of gaseous molecules adsorption on oxide supports. Quantitative InfraRed Thermography Journal. 2011;8:129-37. 